\title{
PeneloPET, a Monte Carlo PET simulation toolkit based on PENELOPE: Features and Validation
}

\author{
S. España ${ }^{1}$, J. L. Herraiz ${ }^{1}$, E. Vicente ${ }^{2}$, J. J. Vaquero ${ }^{2}$, M. Desco ${ }^{2}$, J. M. Udías ${ }^{1}$ \\ ${ }^{1}$ Dpto. Física Atómica, Molecular y Nuclear, Universidad Complutense de Madrid, Spain \\ ${ }^{2}$ Unidad de Medicina y Cirugía Experimental, Hospital GU “Gregorio Marañón”, Madrid, Spain
}

\begin{abstract}
PENELOPE is a Monte Carlo code that simulates the transport in matter of electrons, positrons and photons with energies from a few hundred of $\mathrm{eV}$ to $1 \mathrm{GeV}$. It is robust, fast and very accurate, but it may be unfriendly for people not acquainted with the FORTRAN programming language. We have developed a tookit ('PeneloPET') to prepare simulations of PET and SPECT within PENELOPE. Sophisticated simulations can be setup by modifying just a few simple input files. The output data can be generated at different levels of detail and can be analyzed afterwards with the preferred programming language or tools. In this work, we present the features of PeneloPET as well as validations against other dedicated PET simulation programs and two real scanners.
\end{abstract}

Index Terms - Positron emission tomography, Monte Carlo Simulation

\section{INTRODUCTION}

$\mathrm{P}$ ENELOPE $[1,2]$ is a code for Monte Carlo simulations of the transport in matter of electrons, positrons and photons with energies from a few hundred of $\mathrm{eV}$ to $1 \mathrm{GeV}$. It is robust, fast and very accurate, but it may be unfriendly for people not acquainted with the FORTRAN programming language.

We have developed a toolkit ('PeneloPET') to prepare complete simulations for PET and SPECT with PENELOPE. Sophisticated simulations can be set-up by modifying just a few simple inputs files. The output data can be generated at different levels of post-processing and can be analyzed afterwards with the preferred programming language or tools.

Parameters of the input files may include information about scanner geometry, scintillator material, phantom geometries, shielding, source activity and isotope type. A number of options, such as simulation of positron range, photon noncollinearity and scanner motion can be easily selected from the input file. It has also been implemented the possibility of

Manuscript received November 17, 2006. S. España e-mail: samuel@nuclear.fis.ucm.es; J. L. Herraiz e-mail: joaquin@nuclear.fis.ucm.es. E.Vicente email: evicente@mce.hggm.es, J.J.Vaquero email: juanjo@mce.hggm.es, M. Desco e-mail: desco@mce.hggm.es; J.M.Udias email: jose@nuc2.fis.ucm.es limiting the number and kind of particles involved in the simulation. For instance, it can be chosen to simulate only the initial gamma photons (511 keV and others).

The output files allow for levels of detail. At the lowest one, all the information about each interaction is kept for further analysis. At an intermediate level, just the singles events with the information needed for their analysis is stored. The possibility of pile up and cross talk is taken into account. The third, and highest, level of processing stores the coincidence counts in a compact list-mode file but the information about pile up, scatter, random and selfcoincidence events obtained from the simulation is just summarily available. More elaborated analysis can be made if the user writes his/her own analysis code.

Validation against other PET dedicated codes $[3,4,5,6]$ and two real scanners has been performed, confirming that PeneloPET is a powerful tool for PET research and development, and for the quality assessment of PET images.

To complete the validation, we present measurements of axial sensitivity, radial profiles of coincidences, axial profile of parallel coincidences and central point source sensitivity.

\section{PeneloPeT features}

\section{A Source Code}

PeneloPET is a FORTRAN 77 application based on PENELOPE whose goal is to offer an easy to use toolkit for simulating the physical processes involved in PET. PeneloPET requires minimal time investment for the preparation of the simulation setup and it runs very efficiently, requiring less computational time than analogous tools available.

The source code is composed of two principal modules. The first one is dedicated to the PENELOPE simulation and to the storage of the information for later analysis. The purpose of the second module is to post-process these data, taking into account the Anger logic for positioning the interaction inside the crystal array, pile-up, energy resolution, coincidence time 
window, energy window, time resolution, decay process, dead time, etc.

\section{B Input Files}

Some typical materials for crystals, shielding and phantom are predefined, but PeneloPET allows the definition of new materials. It allows the user to define scanners with rectangular prism blocks of detectors in a ring arrangement, with several axial rings of blocks and layers of crystal in each block.

A few input files have to be defined before starting the simulation. These files contain all the parameters of the setup to be simulated. One file contains general options, in such a way that it is possible to perform the same simulation at different levels of detail regarding the physicals aspects and the post-processing. Another interesting option is the possibility of simulating all the electrons, positrons and photons involved in the experiment or to simulate only the annihilation photons, introducing (or not) positron range and non-collinearity by means of predefined functions. In this way, the computational time can be drastically reduced.

Geometry and material information are easily defined. Scanner parameters and other objects such as shielding and phantoms are included independently. Multiple rings and layers of crystals are allowed.

The activity source is defined separately with its own geometry and the number of simulated events required. When only gamma rays are involved in the simulation, the direction of emission can be restricted, to achieve higher efficiency.

\section{Outputs}

Using the first and basic level of post-processing it is possible to record the information about the track followed for each simulated particle. The built-in PENELOPE visualization tools are fully available in PeneloPET to show the tracks visually. This is especially useful during scanner design phases.

The second level of post-processing gives the information about single events recorded by each block, such as detection time, pile-up, energy, crystal coordinates.

The third a most sophisticated outputs include information about the coincidence events, such as energy and crystal of interaction for each single event, time delay between singles, kind of coincidence (True, Random, Scatter, self-coincidence, etc.)

Many types of histogram are included in PeneloPET to analyze output data and to facilitate the interpretation of the results. Sinograms, singles map, coincidences map, energy spectrum, are other output possibilities.

\section{METHODS FOR VALIDATION}

To perform the validation of PeneloPET we have compared its results with those provided by GATE $[3,4]$ simulations and also with real acquisitions.

a) Axial Profile of Sensitivity: a point source of F-18 is placed in the center of the FOV at several axial positions, measuring the total sensitivity for a specific scanner. To make the comparison easier, low activities were simulated in order to avoid random and pile-up effects.

b) Mouse Phantom: It is rod of $1 \mathrm{~cm}$ diameter filled wil F18 , off-centered in a water cylinder of $2.5 \mathrm{~cm}$ of diameter. For the validation we obtained radial profiles of coincidences from a real acquisition with the rPET scanner [7]. The initial activity was $250 \mu \mathrm{Ci}$ and no energy windows were selected.

c) Ge-68 Annulus: an annulus with an inner diameter of $6.92 \mathrm{~cm}$ and $2 \mathrm{~mm}$ thick is simulated. The activity is $500 \mu \mathrm{Ci}$ and the energy window selected is $100-700 \mathrm{keV}, 250-700 \mathrm{keV}$ and $400-700 \mathrm{keV}$. For this comparison we show profiles of coincidence counts for crystals in the same axial row, acquired with each energy window. The comparison to a real acquisition of the eXplore Vista (GE) scanner [8] is shown.

d) Central Point Source Sensitivity (CPSS): a line source filled with F-18 and placed at the center of the FOV. The source has a length of $6.4 \mathrm{~cm}$, a diameter of $1.5 \mathrm{~mm}$ and an activity of $31 \mu \mathrm{Ci}$. The comparison is performed against a real acquisition of the eXplore Vista (GE) scanner.

\section{RESUlts}

We show here the results of all the simulations than have been performed to validate PeneloPET code.

a ) Axial Sensitivity Profile: First, the number of annihilation events are shown in table 1 . The simulation of a center point source in rPET scanner is between 5 and 6 times faster in PeneloPET than in GATE.

On figure 1 a comparison of the axial sensitivity profiles for several energy window of the rPET scanner is showed. It can be seen that there is some underestimation with PeneloPET compared to GATE ones, probably due to the fact that in GATE simulations X-rays were included in this case. 


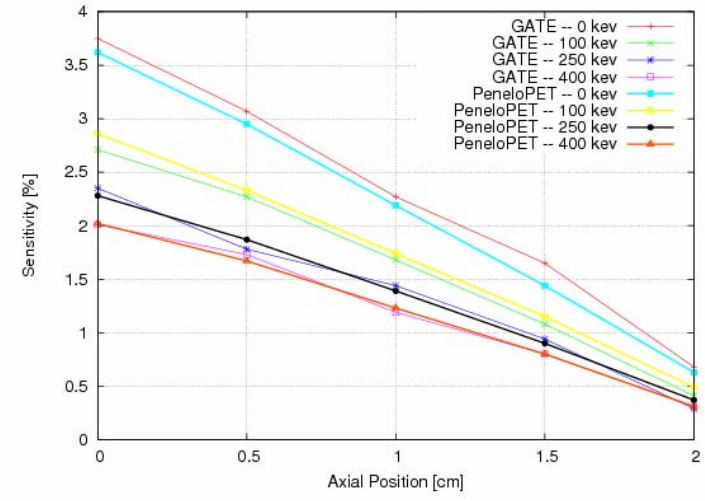

Fig. 1. F-18 point source with low activity in several axial positions. PeneloPET results lie below GATE ones when the lower energy threshold is reduced.

b) Mouse Phantom: Two radial profiles of the sinogram are presented. There is good agreement between real data and simulation in the peak area and also for background counts in the region far from the peak. In the simulation, the contribution to the background from scatter and random coincidences can be identified, and also event coincidences when pile-up takes place in any of the single events.
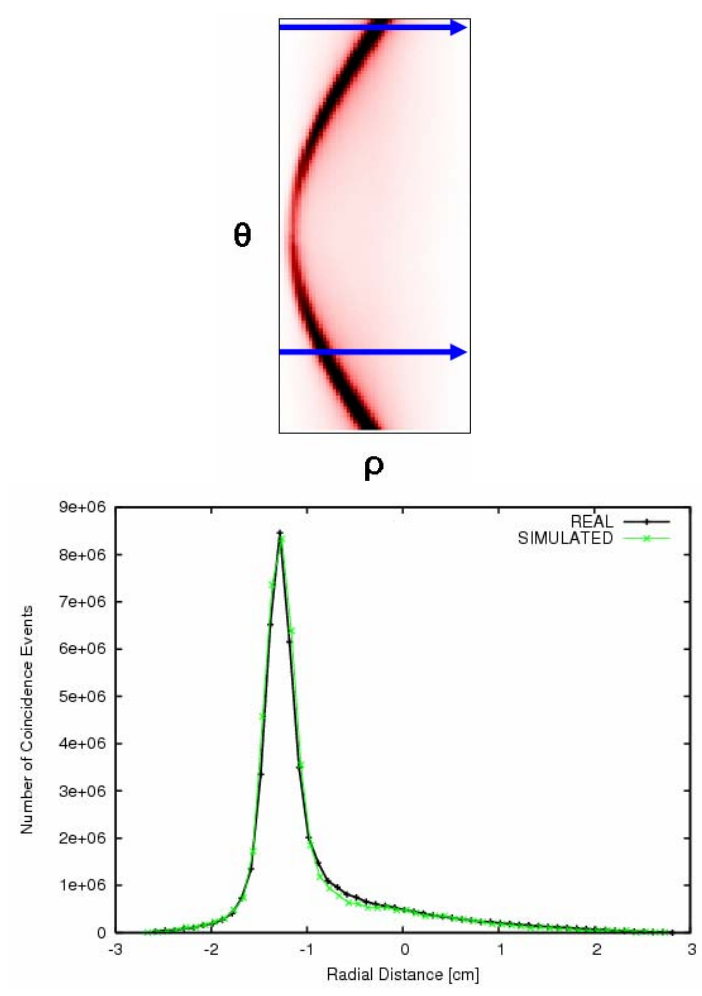

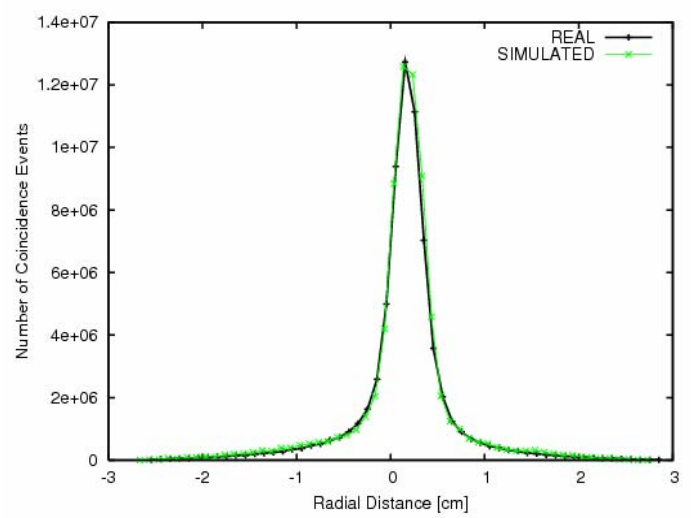

Fig. 2. Water filled cylinder with off-centered rod filled with FDG $(350 \mu \mathrm{Ci})$. Comparison of two radial profiles of the sinogram.

c) Ge-68 Annulus: There is very good agreement between simulation and real data. Large variations of the axial profiles depending on the energy window selected are seen, in agreement with the experimental data. The simulations allow to separate the different contributions in the axial profiles. This is very important to set up a good normalization method that employs high activity reference phantoms. There is a sizeable difference in the fraction of scatter coincidences occurring when the wide energy window $(100-700 \mathrm{keV})$ is chosen, compared to the narrow one (400-700 keV). Random coincidences are more important in the gap region and for the wider energy windows and pile-up and self-coincidences are nearly independent on the energy window, as one would expect.

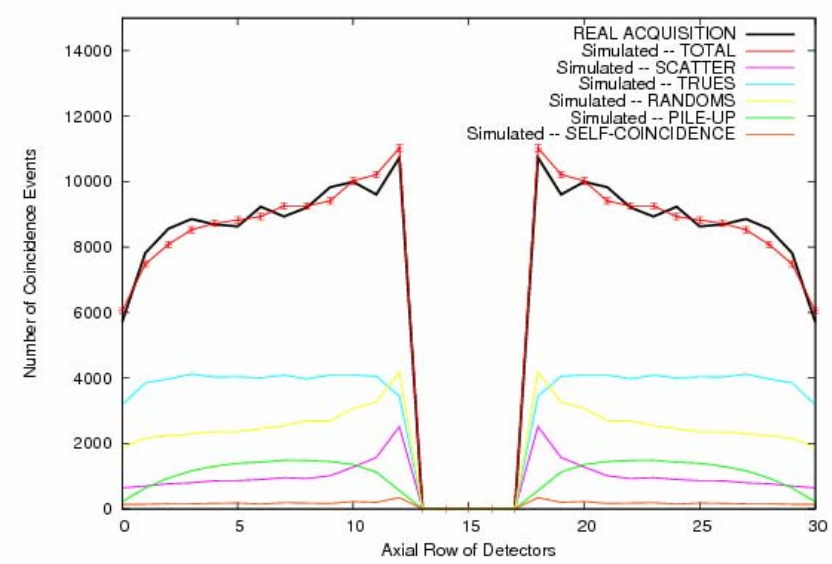



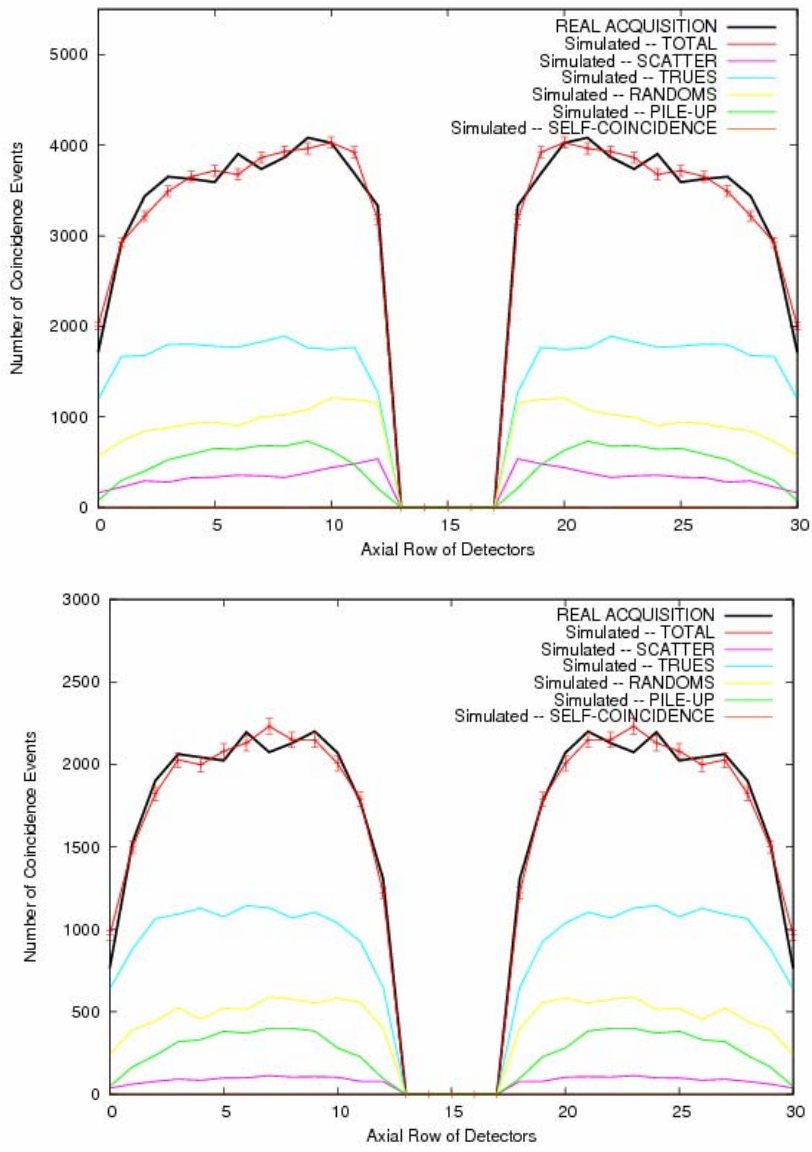

Fig. $3 \mathrm{Ge}-68$ filled plastic annulus for calibration $(500 \mu \mathrm{Ci})$. Comparison of profiles of coincidence counts for crystals in the same axial row. Different energy windows are applied: (Above) 100-700 keV, (Center) $250-700 \mathrm{keV}$, (Below) 400-700 keV.

d) Central Point Source Sensitivity (CPSS): in table 2, good agreement between real and simulated data is shown for the case of the narrow energy window, results are quite sensitive to the energy resolution selected for the simulation.

TABLE I

CENTRAL F-18 POINT SOURCE WITH LOW ACTIVITY FOR RPET SCANNER ONLY $511 \mathrm{KEV}$ PHOTONS ARE SIMULATED. RESULTS FOR AMD ATLHON $3200 \mathrm{MHZ}$ PROCESSOR.

\begin{tabular}{|c|c|c|}
\hline & PeneloPET & GATE \\
\hline Center Point Source & $25000 \mathrm{e}+/ \mathrm{sec}$ & $4000 \mathrm{e}+/ \mathrm{sec}$ \\
\hline Cylinder & $16000 \mathrm{e}+/ \mathrm{sec}$ & --- \\
\hline Annulus & $10000 \mathrm{e}+/ \mathrm{sec}$ & --- \\
\hline
\end{tabular}

On figure 1 a comparison of the axial sensitivity profiles for several energy windows in the rPET scanner is shown. It can be seen that PeneloPET results lie slightly below GATE ones, probably due to that in GATE simulations X-ray contributions were included, but not in PeneloPET.

TABLE II
CPSS: LINE SOURCE FILLED WITH F-18 AND PLACED AT THE CENTRE OF THE FOV. LENGTH $=6.4 \mathrm{CM} ;$ DIAMETER $=15 \mathrm{MM}$. ACTIVITY $=31 \mathrm{MCI}$

\begin{tabular}{|c|c|c|}
\hline & Real Data & PeneloPET \\
\hline $100-700 \mathrm{keV}$ & 5.4 & 5.2 \\
\hline $250-700 \mathrm{keV}$ & 3.6 & 3.6 \\
\hline $400-700 \mathrm{keV}$ & 1.9 & 1.9 \\
\hline
\end{tabular}

Comparison with real data have also been presented. Preliminary results show that single and coincidence rates, and sensitivity profiles obtained with PeneloPET are in good agreement with measured values.

\section{CONCLUSIONS}

PeneloPET is an excellent application for PET and SPECT simulations because of its versatility, speed, and easy-toanalyze outputs. PeneloPET is a tool useful for scanner design, system response calculations, development of random and scatter corrections methods, and many other applications. Validation of the code has been obtained against other tomography-dedicated simulators, and against real data. PeneloPET emerges as a powerful tool to perform PET simulations.

\section{ACKNOWLEDGMENT}

We thanks support from MEC (FPA2006-07393) and CDTEAM (CENIT-Ingenio 2010) Ministerio de Industria, Spain, projects. The numerical computations of this work were done at the "High capacity cluster for physical techniques" of the Faculty for Physical Sciences of the UCM, funded in part by the UE under the FEDER programme and in part by the UCM. Parts of this work were performed under the HGGMUCM contract "Modelización de escáneres PET de alta resolución y reconstrucción estadística de imágenes PET en animales pequeños". J. L. Herráiz also acknowledges support from UCM grant.

\section{REFERENCES}

[1] J. Sempau, E. Acosta, J. Baró, J.M. Fernández-Varea, F. Salvat, “An algorithm for Monte Carlo simulation of coupled electron-photon transport", Nucl. Inst. Meth. In Phy. Res. B 132 (1997) 377-390

[2] F. Salvat, J.M. Fernández-Varea, J. Sempau, "PENELOPE - A Code System for Monte Carlo Simulation of Electron and Photon Transport", OECD, 2003.

[3] S. Jan et al. , "GATE: a simulation toolkit for PET and SPECT". Phys. Med. Biol. 49 (2004) 4543-4561

[4] Lazaro D, Buvat I, Loudos G, Strul D, Santin G, Giokaris N, Donnarieix D, Maigne L, Spanoudaki V, Styliaris S, Staelens S, Breton V. "Validation of the GATE Monte Carlo simulation platform for modelling a CsI(Tl) scintillation camera dedicated to small-animal imaging," Phys. Med. Biol. 49 (2004) 271-285

[5] A. Reilhac, C. Lartizien, N. Costes, S. Sans, Clauden Comtat, R. N. Gunn, and A. C. Evans, "Validation of SORTEO Monte Carlo 
simulations for the geometry of the Concorde MicroPET R4 system" 2004 IEEE Nuc Sci Symp Conf Rec 3036-3039.

[6] A. Reilhac, G. Batan, C. Michel, C. Grova, J. Tohka, D. L. Collins, N. Costes, A. C. Evans, "PET-SORTEO: Validation and Development of Database of Simulated PET Volumes" IEEE Trans on Nuc Sci, Vol. 52, No. 5, (2005) 1321

[7] J. J. Vaquero, E. Lage, L. Ricón, M. Abella, E. Vicente, M. Desco "rPET Detectors Design and Data Processing”, 2005 IEEE Nuc Sci Symp Conf Rec 2885-2889

[8] Y. Wang1, J. Seidel1, B. M.W. Tsui1, J. J. Vaquero, M. G. Pomper "Performance Evaluation of the GE Healthcare eXplore VISTA DualRing Small-Animal PET Scanner” J. of Nuc Med Vol. 47 No. 11 (2006) 1891-1900 